\title{
Proton magnetic resonance neurospectroscopy and EEG cartography in corticobasal degeneration: correlations with neuropsychological signs
}

\author{
J Vion-Dury, N Rochefort, P Michotey, D Planche, M Ceccaldi
}

J Neurol Neurosurg Psychiatry 2004;75:1352-1355. doi: 10.1136/jnnp.2003.018903

\begin{abstract}
Objective: To document the asymmetrical functional brain lesions in corticobasal degeneration (CBD) using proton magnetic resonance neurospectroscopy (MRS) and EEG cartography (EEGq).

Methods: Eight patients with probable CBD were included in the study after full neurological examination and extensive neuropsychological testing, single photon emission computed tomography, anatomical $x$ ray tomodensitometry (TDM), magnetic resonance imaging, and MRS examination.

Results: MR spectra were abnormal in all seven patients in whom the examination could be completed. The EEG was also always modified in the CBD patients, and the abnormalities were enhanced by activation procedures. There was a good correlation between MRS anomalies and clinical presentation, between EEG modifications and neuropsychological patterns, and between metabolic (MRS) impairment and electrophysiological (EEG) slowing.

Conclusions: These results confirm the asymmetrical features of CBD. Combined EEGq/MRS examinations at disease onset and during its subsequent course could provide strong diagnostic evidence of CBD.
\end{abstract}

\footnotetext{
C
} orticobasal degeneration (CBD) is an uncommon degenerative disease characterised by gradual progression of strikingly asymmetrical motor features that are often similar to those of Parkinson's disease. ${ }^{12}$ In addition to extrapyramidal symptoms related to basal ganglia dysfunction, cognitive deficits are frequent. The cognitive signs (aphasia, apraxia, frontal syndrome, visuospatial deficits) also predominantly involve one hemisphere both at the onset and during progression of the disease. Sometimes these signs are the presenting features of the disease. ${ }^{3}$

Conventional electroencephalography (EEG) may be normal at the start of the disease, and often remains unchanged as the disease progresses. In some patients there may be a unilateral slowing, which becomes generalised as the disease evolves. ${ }^{2}$ Brain imaging has shown unilateral atrophy as well as a reduction in glucose metabolism, mainly in the thalamoparietal system and in the frontoparietal areas contralateral to the symptomatology. ${ }^{4}$ A significant and sometimes asymmetrical neuronal loss (low $\mathrm{N}$-acetyl-aspartate signal) has been observed by in vivo proton magnetic resonance spectroscopy (MRS) in various areas, including the centrum semi-ovale, the lentiform nucleus, the putamen, the frontal lobes, and the parietal cortex..$^{5-7}$ Finally, neuropathological examination has demonstrated cortical degeneration in CBD (with histological lesions belonging to the Pick's complex entity) and lesions of the substantia nigra with a diffuse reduction in striatal dopamine concentrations. ${ }^{1}$
Our aims in this study were first, to document the asymmetrical brain degeneration using single voxel neurospectroscopy (MRS) of the parietal cortex (in view of the frequent occurrence of apraxia in CBD) and EEG cartography (EEGq); and second, to evaluate the correlation between the paraclinical variables and clinical (neuropsychological and motor) status.

\section{METHODS \\ Patients}

We studied eight patients (six women, two men, aged 58 to 75 years) meeting the Riley and Lang criteria for CBD. ${ }^{8}$ They presented with mainly cognitive signs, or else their disease had started with cognitive symptoms. The follow up lasted from two to five years.

All the patients were submitted to full conventional neurological examination and extensive neuropsychological testing. A summary of the main clinical and paraclinical data is given in table 1. Quantitative EEG and neurospectroscopy were carried out in, respectively, six and seven of the patients. Five patients had both examinations.

\section{MR spectroscopy}

Proton single voxel MRS was done using a Siemens Symphony Quantum system (1.5 T), during the standard MRI procedure. For all data acquisition and processing we used fully automated Singo VAl5 software. Spectra were recorded bilaterally from the superior parietal region using a $135 \mathrm{~ms}$ point resolved spectroscopy (PRESS) sequence (128 summations). The spectroscopic volume of interest (VOI) was $15 \times 15 \times 15 \mathrm{~mm}$. The areas of $\mathrm{N}$-acetyl-aspartate (NAA), creatine-phosphocreatine ( $\mathrm{Cr}$ - $\mathrm{PCr}$ ), and choline (Cho) signals were determined using automatic processing procedures. The following metabolic ratios were then calculated: NAA/Cho, $\mathrm{NAA} / \mathrm{Cr}-\mathrm{PCr}$, and Cho/Cr-PCr. A spectrum was considered abnormal when one of the calculated metabolic peak ratio values was lower or higher than the lowest or highest value found in our control population in the same region of interest. In the control population, the NAA, Cho, or Cr-PCr signal asymmetry between spectra recorded in the two parietal regions was less than $10 \%$.

\section{Electroencephalography}

Conventional EEG was undertaken in six patients using a Brainlab-Schwartzer digital apparatus with 19 channels. Sample frequency was $250 \mathrm{~Hz}$ and electrode impedance was kept below $5 \mathrm{k} \Omega$. Spectral analysis was conducted on the signal coming from monopolar derivations (versus a common reference) in the following conditions: resting basal with eyes

Abbreviations: $C B D$, corticobasal degeneration; $E E G q, E E G$ cartography; MRI, magnetic resonance imaging; MRS, proton magnetic resonance neurospectroscopy; TDM, tomodensitometry 


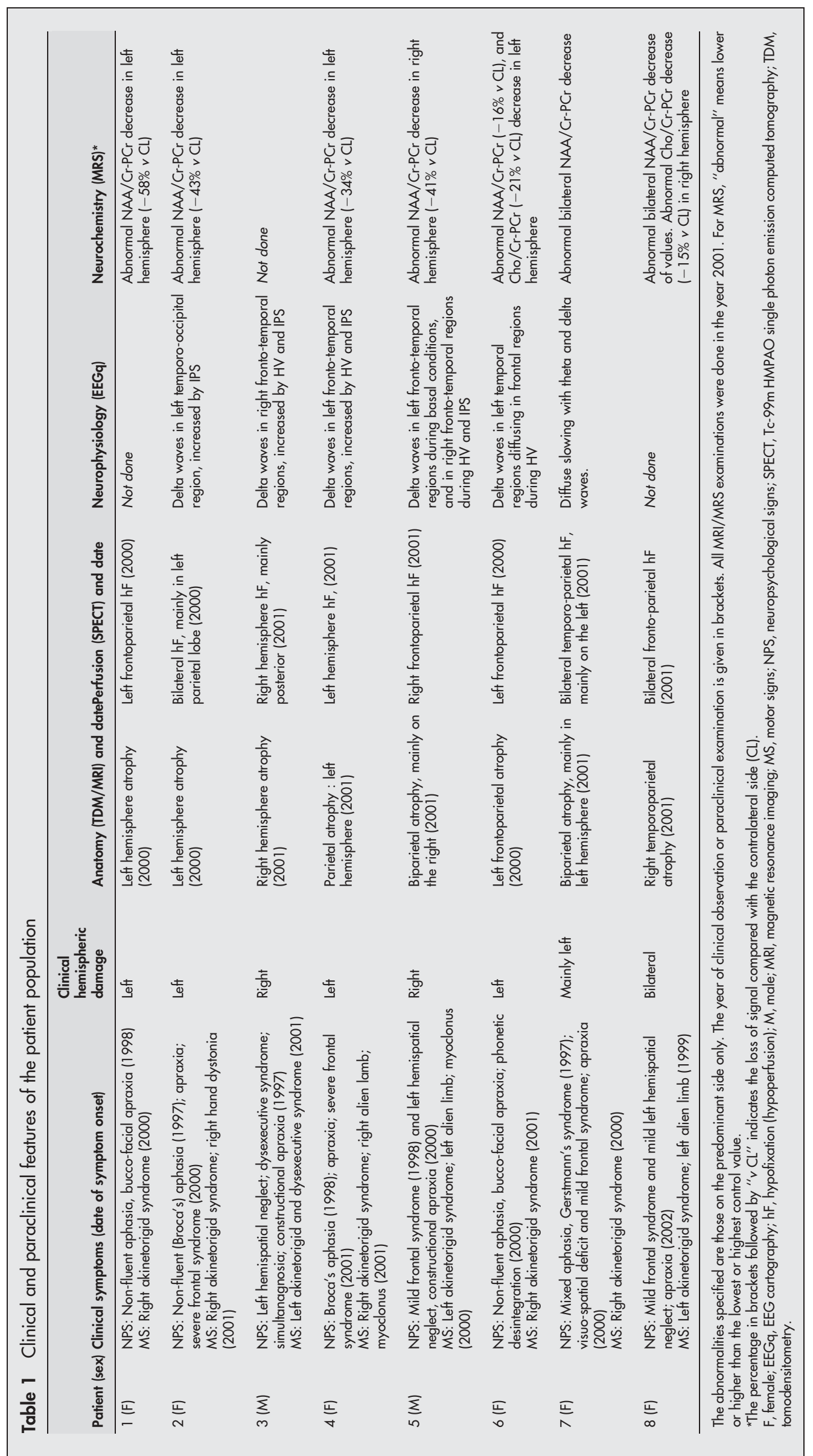




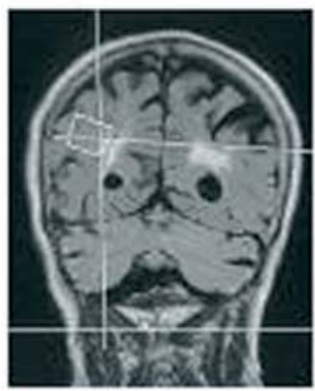

Left parietal lobe

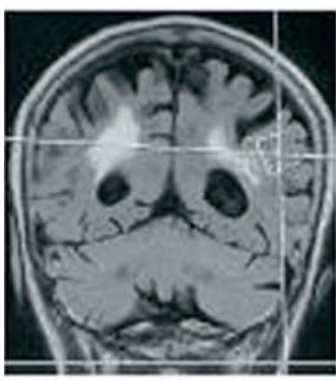

MRI

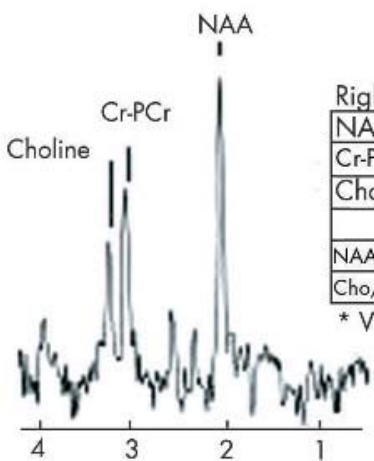

PPM

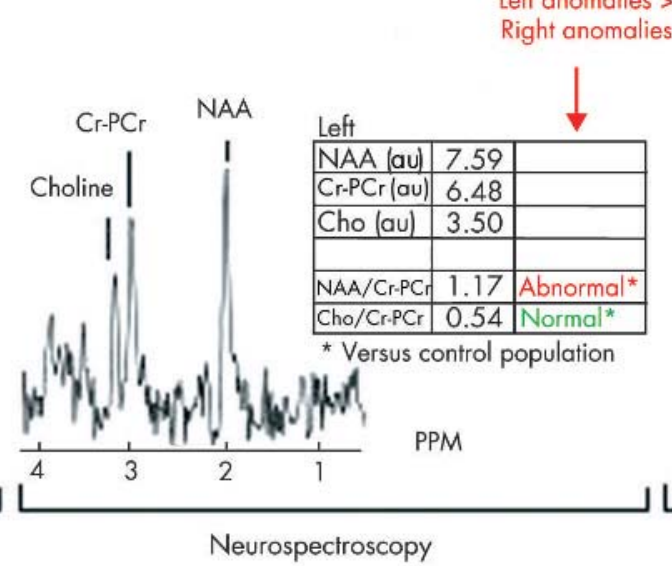

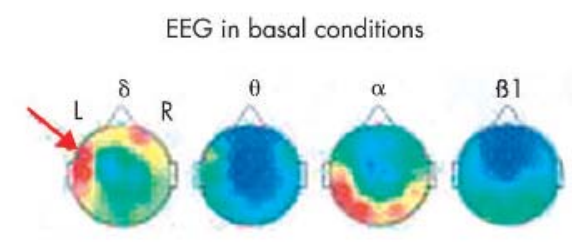

EEG after hyperventilation
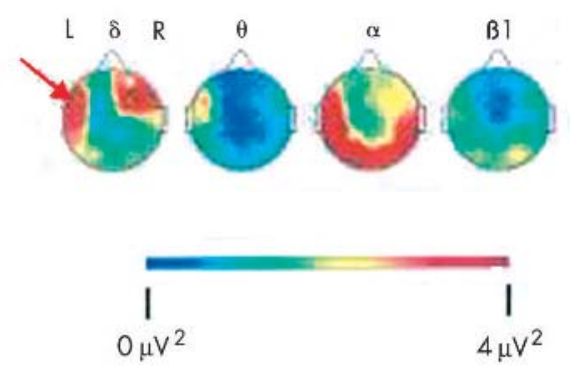

Quantitative EEG

Figure 1 Results of magnetic resonance imaging (MRI), proton magnetic resonance neurospectroscopy, and EEG cartography (EEGq) in patient No 6. EEGq is presented as absolute maps obtained for each frequency band. au, absolute units; Cho, choline; $\mathrm{Cr}$, creatine; $\mathrm{NAA}, \mathrm{N}$-acetyl-aspartate; $\mathrm{PCr}$, phosphocreatine; PPM, parts per million.

closed and still; hyperventilation; and intermittent photic stimulation. Each signal, analysed by Fourier transform, was constituted by summation of several two-second epochs in a stationary electrophysiological state, in the absence of artefact, corresponding to a signal duration varying from 12 to 30 seconds. The absolute power spectrum, calculated under each electrode (in $\mu \mathrm{V}^{2}$ ) with a frequency resolution of $0.5 \mathrm{~Hz}$, was carefully analysed. The absolute power spectral maps were then displayed. In these maps, the mean spectral power was calculated for the beta $(13-25 \mathrm{~Hz})$, alpha $(8-13 \mathrm{~Hz})$, theta $(5-7 \mathrm{~Hz})$, and delta $(1-4 \mathrm{~Hz})$ frequency bands. All the spectral maps were calibrated from the absolute spectral power scale obtained in resting conditions.

\section{RESULTS AND DISCUSSION \\ Patient population}

We observe first, that all the patients in our population except one (No 8) presented with neuropsychological symptoms predominantly involving one hemisphere (aphasia, left hemisphere: patients 1, 2, 4, 6 7; visuospatial deficits, right hemisphere: patients 3 and 5); second, that in all the patients the motor signs detected during disease evolution and the neuropsychological evaluations were congruent with the MRS and EEGq results, indicating lateralised hemispheric damage (a right extrapyramidal syndrome with aphasia and a left extrapyramidal syndrome with visuospatial deficit); and third, that at the time of the MRS/EEGq examination, the clinical presentation remained clearly asymmetrical in seven patients, in agreement with TDM and SPECT results. In the remaining patient (No 7), the asymmetry was ambiguous.

\section{MRS}

Typical magnetic resonance spectra from a patient are shown in fig 1. In both spectra in this patient, we can see a significant decrease in the NAA/Cr-PCr ratio, more pronounced on the left, and marked asymmetry of the NAA signal, which is reduced by $26 \%$ on the left side compared with the right.

MRS was found to be abnormal in seven patients, and the metabolic asymmetry was more than $20 \%$ in five of these (table 1). All patients showed an abnormal decrease in the NAA/Cr-PCr ratio, and two of them had a disturbance of the Cho/Cr-PCr ratio. MRS correlated with NPS in all the patients, whatever their clinical presentation.

These results are in agreement with previous studies ${ }^{5-7}$ showing metabolic impairment in all patients, and metabolic asymmetry when asymmetry of clinical symptoms was present. The frequent and substantial reduction of the $\mathrm{NAA} / \mathrm{Cho}$ or NAA/Cr-PCr ratios, or both, pinpoint the severe neuronal damage in CBD. The reduction in the choline signal in two cases (Nos 6 and 8) is also an interesting finding related to a possible membrane defect. Such a membrane defect might be related to a global decrease in the enzymatic activity of Kennedy's pathway, as a part of the asymmetrical hypometabolism observed in $\mathrm{CBD}^{12}$ (Kennedy's pathway is the pathway for phosphatidylcholine, and then phospholipid synthesis from choline, ATP, and glycerol ${ }^{9}$ ). This hypothesis seems more probable than a reduction in brain acetylcholine content because, first, such a reduction has not been documented in CBD (in contrast to Alzheimer's disease), and second, an asymmetrical reduction in striatal dopamine has been found. ${ }^{1}$ 


\section{Electroencephalography}

The EEG cartography from patient No 6 is shown in fig 1 under resting and hyperventilation conditions. In the six patients examined (table 1), the EEG recording shows an alpha rhythm that is commonly present, and also the occurrence EEG abnormalities (generally enhanced by hyperventilation or intermittent photic stimulation) such as slowing and sometimes sharp waves in the delta or theta frequency range. These abnormalities were lateralised in five patients (in one after hyperventilation) and were bilateral in one. In our population, EEGq was always found to be abnormal, and the abnormalities were markedly enhanced by activation procedures such as hyperventilation. The location of the neurophysiological abnormalities detected using EEGq was in agreement with MRI, SPECT, and neuropsychological tests.

Our results contrast with those of Lu et al, ${ }^{4}$ Rebeiz et al, ${ }^{10}$ and Riley et $a l^{18}$ who have often found no significant modification of brain electrical activity in CBD. We can think of two possible explanations for these discrepancies: first, our patients may have had more advanced disease, and second, spectral analysis is more sensitive than standard visual inspection for detecting anomalies.

Finally, the asymmetrical EEGq pattern observed contrasts with the findings in Parkinson's disease, in which the EEG is often described as normal, becoming abnormal (with diffuse slowing) only in the most advanced cases, particularly in patients with pronounced akinesia. ${ }^{11}$

\section{Correlations between neurochemistry and electrophysiology}

In the five patients who had both examinations, the EEG findings were correlated with the MR spectroscopy and the clinical and paraclinical examinations (in patients Nos 2, 4, 6, and 7); in patient No 5, the correlation was only obtained with hyperventilation and intermittent photic stimulation.

\section{Conclusions}

It is of interest to observe that in this degenerative disease, slowing of EEG activity correlates with a decrease in NAA. The marked correlation between the EEG findings and MRS in this asymmetrical disease when it becomes secondarily generalised forms a strong argument for an integrated concept of the brain pathology based on electrophysiology (classically) and neurochemistry. The pronounced decrease in this neuronal marker in the parietal regions, as well as the intense modification of the neuronal dynamics detected by EEGq, suggests significant and extended cortical degeneration. This pattern of neuronal loss or damage in CBD is probably different in topography, extent, and intensity from that found in Parkinson's disease. This could explain the extreme variability of the MRS results observed in Parkinson's disease, ${ }^{12}$ in agreement with the neurophysiological features.
Further investigations will be undertaken to confirm the presence of unilateral degeneration in the early stages of CBD and the persistence of an asymmetrical pattern of degeneration during the course of the disease based on the metabolic and neurophysiological abnormalities.

Our study suggests that a simple strategy combining MRI with two neurospectroscopic acquisitions and quantitated standard EEG with careful spectral analysis could be a useful diagnostic tool for CBD in the early stages of the disease.

\section{ACKNOWLEDGEMENTS}

The MRS studies was supported by Fondation Hôpital Ambroise Paré. We thanks Dr M Pierlovisi for her fruitful remarks about electrophysiology, and the technicians of the Departments of Clinical Neurophysiology and of Radiology.

\section{Authors' affiliations}

J Vion-Dury, D Planche, Service de Neurophysiologie Clinique, Hôpital de La Conception, Marseille, France

N Rochefort, M Ceccaldi, Service de Neurologie et Neuropsychologie, Hôpital de La Timone, Marseille

P Michotey, Service de Radiologie, Hôpital Ambroise Paré, Marseille

Competing interests: none declared

Correspondence to: Dr J Vion-Dury, Service de Neurophysiologie Clinique, Hôpital de La Conception, 13005 Marseille, France; jvion-dury@ap-hm.fr

Received 16 May 2003

In revised form 24 November 2003

Accepted 29 November 2003

\section{REFERENCES}

1 Riley $\mathrm{DE}$, Lang $A E$, Lewis $A$, et al. Cortical-basal ganglionic degeneration. Neurology 1990;40:1203-12.

2 Ozsancak C, Auzou P, Hannequin D. La dégénérescence corticobasale. Rev Neurol 1999;155:1007-20.

3 Kertesz A, Martinez-Lage P, Davidson W, et al. The corticobasal degeneration syndrome overlaps progressive aphasia and fronto-temporal dementia. Neurology 2000;55:1368-75.

4 Lu CS, Ikeda, Terada K, et al. Electrophysiological studies of early stage corticobasal degeneration. Mov Disord 1998;13:140-6.

5 Tedeschi G, Litvan I, Bonavita S, et al. Proton magnetic resonance spectroscopic imaging in progressive supranuclear palsy, Parkinson's disease and corticobasal degeneration. Brain 1997;120:1541-52.

6 Nakase T, Mitzuno T, Takanashiu Y, et al. A lateralized reduction of NAA in a case of corticobasal degeneration (CBD): application of proton magnetic resonance spectroscopy. No To Shinkei 1998;50:421-5.

7 Abe K, Terakawa H, Takanashi $M$, et al. Proton magnetic resonance spectroscopy of patients with parkinsonism. Brain Res Bull 2000;52:589-95.

8 Riley DE, Lang AE. Clinical diagnostic criteria of corticobasal degeneration. Adv Neurol 2000;82:1233-45.

9 Klein J, Köppen A, Löffelholz K, et al. Uptake and metabolism of choline by rat brain after acute choline administration. J Neurochem 1992;58:870-6.

10 Rebeiz JJ, Kolodny EH, Richardson EP. Cortico-dentatonigral degeneration with neuronal achromasia. Arch Neurol 1968;18:20-33.

11 Niedermeyer E, Lopes da Silva F. Electroencephalography. Basic principles, clinical applications, and related fields. Philadelphia: Lippincott Williams and Wilkins, 1999:354.

12 Clarke CE, Lowry M. Systematic review of proton magnetic resonance spectroscopy of the striatum in parkinsonian syndromes. Eur J Neurol 2001;8:573-7. 